\section{BMJ Open} Ophthalmology

\title{
Comparison of the Kane formula with existing formulas for intraocular lens power selection
}

\author{
Benjamin J Connell, ${ }^{\oplus 1}$ Jack X Kane ${ }^{2}$
}

To cite: Connell BJ, Kane JX. Comparison of the Kane formula with existing formulas for intraocular lens power selection. BMJ Open Ophthalmology 2019;4:e000251. doi:10.1136/ bmjophth-2018-000251

Received 16 November 2018 Revised 27 February 2019 Accepted 2 March 2019
Check for updates

(c) Author(s) (or their employer(s)) 2019. Re-use permitted under CC BY-NC. No commercial re-use. See rights and permissions. Published by BMJ.

${ }^{1}$ Eye Surgery Associates, Melbourne, Victoria, Australia ${ }^{2}$ Royal Victorian Eye and Ear Hospital, Melbourne, Victoria, Australia

Correspondence to Dr Benjamin J Connell; benconnell@outlook.com.au

\section{ABSTRACT}

Objective To compare the accuracy of a new intraocular lens (IOL) power formula (Kane formula) with existing formulas using IOLMaster, predominantly model 3 , biometry (measures variables axial length, keratometry and anterior chamber depth) and optimised lens constants. To compare the accuracy of three new or updated IOL power formulas (Kane, Hill-RBF V.2.0 and Holladay 2 with new axial length adjustment) compared with existing formulas (OIsen, Barrett Universal 2, Haigis, Holladay 1 , Hoffer Q, SRK/T).

Methods and analysis A single surgeon retrospective case review was performed from patients having uneventful cataract surgery with Acrysof IQ SN60WF IOL implantation over 11 years in a Melbourne private practice. Using optimised lens constants, the predicted refractive outcome for each formula was calculated for each patient. This was compared with the actual refractive outcome to give the prediction error. Eyes were separated into subgroups based on axial length as follows: short $(\leq 22.0 \mathrm{~mm})$, medium $(>22.0$ to $<26.0 \mathrm{~mm})$ and long $(\geq 26.0$ $\mathrm{mm})$.

Results The study included 846 patients. Over the entire axial length range, the Kane formula had the lowest mean absolute prediction error $(\mathrm{p}<0.001$, all formulas). The mean postoperative difference from intended outcome for the Kane formula was $-0.14+0.27 \times 1(95 \% \mathrm{LCL}$ $-1.52+0.93 \times 43 ; 95 \%$ UCL $+0.54+1.03 \times 149)$. The formula demonstrated the lowest absolute error in the medium axial length range $(p<0.001)$. In the short and long axial length groups, no formula demonstrated a significantly lower absolute mean prediction error. Conclusion Using three variables (AL, $K, A C D$ ), the Kane formula was a more accurate predictor of actual postoperative refraction than the other formulae under investigation. There were not enough eyes of short or long axial length to adequately power statistical comparisons within axial length subgroups.

\section{INTRODUCTION}

Refractive expectations following cataract surgery are increasing. Modern biometry, newer intraocular lens (IOL) formulas and surgical techniques have all contributed to an improvement in refractive outcome prediction accuracy. In 2011, a large series reported $71 \%$ and $95 \%$ of cases were within 0.5 and $1.0 \mathrm{D}$ of predicted refraction. ${ }^{1}$ In comparison,

\section{Key messages}

What is already known about this subject?

- Modern intraocular lens (IOL) formulas have provided unprecedented accuracy.

There are no publications assessing the new Kane formula and the RBF/Holladay 2 formulas following recent modifications.

What are the new findings?

- The Kane formula provided improved accuracy over widely used modern formulas.

- There was no increase in relative accuracy of the RBF and Holladay 2 formulas following their modification.

How might these results change the focus of research or clinical practice?

Use of the Kane formula should be given strong consideration in IOL selection.

a more recent large multicentre study reported up to $81 \%$ and $98 \%$ of eyes within 0.5 and $1.0 \mathrm{D} .^{2}$

Older generation formulas: Haigis, Hoffer Q, Holladay 1 and SRK/T are used less frequently as more modern formulas exhibiting greater accuracy are now available. With incorporation into biometers, these formulas are more accessible and less prone to transcription errors when compared with those accessed via stand-alone software or the internet. Recent studies reporting large series $^{2}{ }^{3}$ have demonstrated the Barrett Universal II formula to have the lowest absolute error compared with other modern formulas.

The Hill-RBF method uses adaptive learning from a large dataset to predict refractive outcomes. For a given eye, it relies on adequate numbers of eyes of a similar dimension to provide an accurate prediction. If the dataset has insufficient number of eyes of a similar dimension, an out of bounds message is provided. Kane et $a t^{t}$ demonstrated the Hill-RBF V.1 formula had a higher mean absolute prediction error when compared with the Barrett Universal II formula. Following 
Table 1 Indications for subject exclusion from the analysis

\begin{tabular}{lc}
\hline Parameter & Eyes (n) \\
\hline Previous corneal surgery (PK, LVC, RK or PTK) & 14 \\
$\begin{array}{l}\text { Other corneal disease (KCN, ectasia, PMD, } \\
\text { corneal scars) }\end{array}$ & 15 \\
CDVA less than 6/12 & 23 \\
No postoperative refraction & 58 \\
$\begin{array}{l}\text { Incomplete biometry or out of range of any } \\
\text { formula }\end{array}$ & 27 \\
Both eyes operated on (random exclusion) & 419 \\
\hline
\end{tabular}

$\mathrm{KCN}$, keratoconus; LVC, laser vision correction; $\mathrm{PK}$, penetrating keratoplasty; PMD, pellucid marginal degeneration; PTK, phototherapeutic keratectomy; RK, radial keratotomy.

modifications, a new V.2 of the Hill-RBF formula (hereafter referred to as the Hill 2.0) has now been released, derived from a larger dataset reportedly making an out of bounds scenario less likely. The Holladay 2 formula has also been updated to include a new axial length adjustment using data from Melles $e t a l^{2}{ }^{2}$ The Kane formula is a new IOL power formula created using several large data sets from selected high-volume surgeons that uses a combination of theoretical optics, thin lens formulas and 'big data' techniques to make its predictions. The Kane formula uses the axial length, keratometry, anterior chamber depth, lens thickness, central corneal thickness and gender of the patient to make its predictions.

To our knowledge, no currently published study has assessed the Hill 2.0, Kane or Holladay 2 with new axial length adjustment.

\section{MATERIALS AND METHODS}

This study was performed on patients in the Author's (BC) private practice in Melbourne, Australia with ethics approval obtained from the local ethics committee.

Inclusion criteria were patients 18 years and over having uncomplicated conventional or femtosecond laser assisted cataract surgery performed by a single surgeon (BC) at a private operating facility. Capsulotomies were

\begin{tabular}{|c|c|}
\hline Parameter & Value: mean \pm SD (range) \\
\hline Axial length & $23.70 \pm 1.28(20.49-31.77)$ \\
\hline Anterior chamber depth & $3.12 \pm 0.39(2.01-4.36)$ \\
\hline Mean keratometry & $43.78 \pm 1.48(39.65-48.12)$ \\
\hline Flat keratometry & $43.45 \pm 1.49(38.05-47.64)$ \\
\hline Steep keratometry & $44.11 \pm 1.53(39.93-50.37)$ \\
\hline Lens thickness & $4.56 \pm 0.45(3.52-5.75)$ \\
\hline IOL power & $21.2 \pm 3.4(6.0-30.0)$ \\
\hline Age & $77.8 \pm 9.58$ \\
\hline Gender distribution & 364 male; 500 female \\
\hline
\end{tabular}

$\mathrm{IOL}$, intraocular lens.

\begin{tabular}{|c|c|c|}
\hline Formula & Lens constant & Optimised constant \\
\hline Barrett Universal & Lens factor & 118.75 \\
\hline Haigis & $\mathrm{a} 0, \mathrm{a} 1, \mathrm{a} 2$ & $-0.946,0.234,0.217$ \\
\hline Hill 2.0 & A constant & 118.69 \\
\hline Hoffer Q & $A C D$ & 5.457 \\
\hline Holladay 1 & Surgeon factor & 1.68 \\
\hline Holladay 2 & ACD & 5.345 \\
\hline Kane & A constant & 118.73 \\
\hline Olsen & ACD & 4.47 \\
\hline $\mathrm{SRK} / \mathrm{T}$ & A constant & 119.05 \\
\hline
\end{tabular}

centred on the pupil with implantation of an Acrysof IQ SN60WF (Alcon Laboratories) inserted through a 2.4 $\mathrm{mm}$ clear corneal incision.

Exclusion criteria were factors that might impact the postoperative refractive outcome which included: (1) preoperative comorbidities (significant corneal scarring, keratoconus or other ectasia, keratoplasty, past laser vision correction, corneal relaxing incisions), (2) intraoperative complication (anterior or posterior capsule tear, vitreous prolapse or zonular dehiscence), (3) postoperative complications (persistent corneal oedema) or (4) postoperative corrected distance visual acuity worse than $6 / 12$, refraction performed before day 21 postoperatively or incomplete documentation. If both eyes of one patient met the inclusion criteria, one eye was randomly chosen for inclusion.

Preoperative biometry was performed using the IOLMaster biometer models 3, 500 or 700 (software 3.2, 7.5 and 1.50 , respectively). Postoperative refraction was performed by an orthoptist. For analysis, values were transcribed manually from the patient electronic medical record into an Excel spreadsheet (Microsoft, Redmond, Washington, USA). Lens thickness and central corneal thickness were only available where biometry was performed with IOLMaster 700 model as the earlier models did not measure these parameters.

The results for the Kane were provided by the author (Kane) prior to the calculation of the other formulas. Predictions for the Haigis, ${ }^{5}$ Hoffer $Q{ }^{6}{ }^{6}$ Holladay $1^{8}$ and $\mathrm{SRK} / \mathrm{T}^{9}$ formulas were obtained using a previously validated $^{3}$ excel spreadsheet, programmed using the original publications and errata. Calculated results were validated against biometer printouts. Data were entered into the respective third-party calculators for the other formulas: PhacoOptics programme for the Olsen formula, ${ }^{10}$ IOL Consultant software for the Holladay 2 formula, ${ }^{11}$ and online calculators for the Barrett Universal $2^{12}$ and the Hill 2.0. ${ }^{13}$ If the refractive aim was outside the limit of the Hill 2.0, then the refractive result was extrapolated.

The constant for each formula was optimised to produce a mean prediction error (ME) of zero (or as close as possible) by performing multiple iterations of 


\begin{tabular}{|c|c|c|c|c|c|c|c|}
\hline Formula & MAE & ME & STDEV & MedAE & $\pm 0.25 \mathrm{D}$ & $\pm 0.50 \mathrm{D}$ & $\pm 1.00 \mathrm{D}$ \\
\hline Kane & 0.329 & 0.00 & 0.445 & 0.231 & 52.4 & 77.9 & 96.6 \\
\hline Olsen & 0.342 & 0.00 & 0.460 & 0.264 & 47.9 & 77.2 & 96.1 \\
\hline Hill 2.0 & 0.346 & 0.00 & 0.463 & 0.264 & 48.3 & 75.3 & 96.4 \\
\hline Barrett & 0.350 & 0.00 & 0.469 & 0.268 & 47.9 & 75.2 & 96.4 \\
\hline Holladay 2 & 0.357 & 0.00 & 0.477 & 0.273 & 46.8 & 76.0 & 95.5 \\
\hline Haigis & 0.358 & 0.00 & 0.480 & 0.271 & 45.5 & 75.5 & 96.1 \\
\hline Holladay 1 & 0.358 & 0.00 & 0.475 & 0.276 & 45.1 & 73.4 & 96.1 \\
\hline $\mathrm{SRK} / \mathrm{T}$ & 0.369 & 0.00 & 0.489 & 0.293 & 44.7 & 72.1 & 95.9 \\
\hline Hoffer Q & 0.381 & 0.00 & 0.499 & 0.311 & 40.3 & 73.6 & 95.5 \\
\hline
\end{tabular}

MAE, mean absolute prediction error; ME, mean prediction error; MedAE, median absolute prediction error; ST DEV, SD deviation of the prediction error.

the data using varying constants. For the Haigis formula, only the a 0 constant was optimised.

For some formulas, a ME of zero could not be obtained due to limitations in how many decimal places could be entered for the constant into the calculator. In these cases, the small residual mean error was removed by adjusting the refractive prediction error for each eye by an amount equal to the ME in that group as described in the JCRS editorial by Wang et al. ${ }^{14}$

The predicted postoperative refraction for each patient was calculated using the optimised IOL constants. The prediction error was then calculated as the actual postoperative refraction minus the refractive result predicted by each formula.

The mean absolute error (MAE), median absolute prediction error (MedAE), ME and SD of the prediction error (STDEV) as well as the percentage of eyes that had a prediction error within $\pm 0.25,0.50$ and $1.00 \mathrm{D}$ were calculated for each formula. Eyes were separated into subgroups based on axial length as follows: short $(\leq 22.0$ $\mathrm{mm}$ ), medium $(>22.0$ to $<26.0 \mathrm{~mm})$ and long $(\geq 26.0$ $\mathrm{mm})$.

The differences in absolute error between formulas were assessed using the Friedman test. In the event of a significant result, posthoc analysis was undertaken using the Wilcoxon signed-ranks tests for pairwise comparisons with Bonferroni correction as suggested by Aristodemou et $a l^{15} \mathrm{~A} \mathrm{p}$ value of less than 0.05 was considered significant. Statistical analysis was performed using R (R Project, R Foundation, Vienna, Austria).

\section{RESULTS}

Of the 1402 patients having cataract surgery with implantation of the Alcon SN60WF IOL, 556 were excluded (see table 1 for reasons and number), leaving 846 patients eligible for inclusion in the study (see table 2 for characteristics of included eyes). Optimised constants are shown in table 3.

Over the entire axial length range, a statistically significant difference existed (see table 4) between the formulas $(\mathrm{p}<0.001)$. The Kane had a significantly lower absolute error $(0.329)$ than all other IOL formulas $(\mathrm{p}<0.001)$.

The Olsen formula had the next lowest MAE (0.342), significantly lower than the remaining formulas $(\mathrm{p}<0.05)$ except compared with the Hill 2.0 (0.346) and the Barrett Universal 2 (0.350). Of the earlier generation formulas, Holladay 1 (0.358) and Haigis (0.358) had the lowest absolute error, significantly lower than Hoffer $Q(0.381)$.

The Hill 2.0 provides an out of bounds statement when the biometry parameters are outside of the range of defined accuracy. The Hill 2.0 provided an out of bounds warning in $11.5 \%$ of cases. Interestingly, the MAE for the Hill 2.0 was 0.347 for in bound cases compared with 0.341 for out of bounds cases.

Table 5 describes the intended, postoperative and difference between the two for the different formula in spherocylinder form as described in the paper by Aristodemou et $\mathrm{l}^{16}$ In the short axial length eyes group $(\mathrm{n}=46)$, there was no statistically significant difference between formula $(p=0.16)$. No posthoc analysis was performed because of the lack of statistically significant result (table 6).

In the Medium axial length eyes group $(n=774)$, there was a statistically significant difference between groups $(p<0.01)$. The Kane formula was more accurate than all others $(p<0.01$ in all cases). There was no difference between the Barrett, Hill 2.0, Olsen and Holladay 1 formulas.

In the long axial length eyes group $(n=44)$, there was a statistically significant difference $(\mathrm{p}<0.01)$ between groups. The most accurate formulas were Haigis, Kane, Barrett, Holladay 2, Olsen and Hill 2.0; however, there were no significant differences within this group. All were significantly more accurate than the earlier generation formulas: SRK/T, Hoffer $Q$ and Holladay $1(\mathrm{p}<0.05)$.

No statistically significant difference in accuracy between the different IOLMaster biometers was noted. For the Haigis formula, the median absolute error for subjects having their biometry with Model 3 was 0.275 (IQR: 0.128-0.492), Model 500: 0.374 (0.198-0.631) 
Table 5 Intended, postoperative and difference between the intended and postoperative refractive error

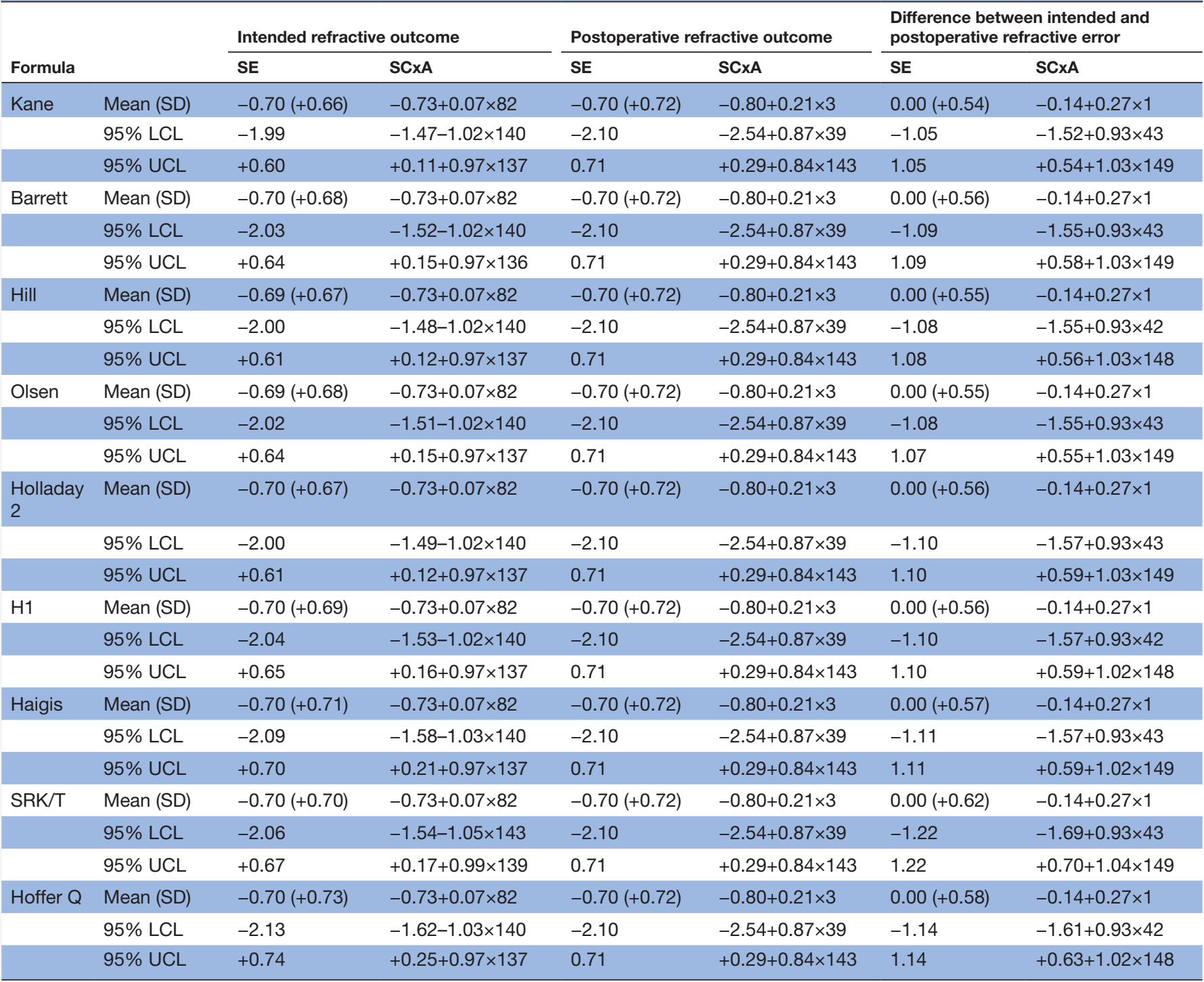

Spherical equivalent (SE), Compound refractive error (SCXA), SD, Lower confidence limit (LCL), Upper confidence limit. LCL, lower confidence limit ; SCXA, compound refractive error; SE, spherical equivalent ; UCL, upper confidence limit.

\begin{tabular}{llll}
\hline \multicolumn{3}{l}{$\begin{array}{l}\text { Table } 6 \\
\text { length }\end{array}$} & \multicolumn{3}{l}{ Mean absolute error for each formula by axial } \\
\hline Formula & Short & Medium & Long \\
\hline Kane & 0.441 & 0.322 & 0.326 \\
\hline Olsen & 0.442 & 0.336 & 0.352 \\
\hline Hill 2.0 & 0.440 & 0.340 & 0.358 \\
\hline Haigis & 0.472 & 0.353 & 0.322 \\
\hline H1 & 0.438 & 0.343 & 0.545 \\
\hline Barrett & 0.479 & 0.344 & 0.331 \\
\hline Holladay 2 & 0.483 & 0.350 & 0.348 \\
\hline SRK/T & 0.475 & 0.360 & 0.407 \\
HofferQ & 0.476 & 0.368 & 0.511 \\
\hline
\end{tabular}

and Model 700: $0.236(0.120-0.468)$. Mood's median test demonstrated no significant difference $\chi^{2}(2)=5.59$, $\mathrm{p}=0.061$.

\section{DISCUSSION}

The Kane formula had the most accurate outcomes with the lowest MAE, STDEV, MedAE and highest percentage of eyes within $0.25,0.50$ and $1.00 \mathrm{D}$ prediction errors. No other published studies have assessed this formula meaning we are unable to compare our results to other papers.

The Olsen formula had the next lowest MAE which was significantly lower compared with all other formulas except the Hill 2.0. In a study by Cooke $e t a l,{ }^{17}$ the PhacoOptics version of the Olsen formula (Olsen PO) showed the least accurate result of the formulas assessed 
when using the IOLMaster biometer but strangely the most accurate when using the Lenstar biometer. Our study showed the Olsen PO formula to have the second lowest MAE out of the formulas assessed indicating the Olsen PO formula may perform well using the IOLMaster biometer.

The largest study to assess the Hill-RBF showed it to have a higher MAE than the Barrett Universal 2, Holladay 1 and SRK/T formulas. ${ }^{4}$ The updated version of the Hill-RBF performed better in our study outperforming each of these formulas although the increased accuracy compared with the Barrett was not statistically significant. Although the Hill-RBF website reports an out of bounds 'will be less common', in our study $11.5 \%$ of calculations returned an 'Out of Bounds' prediction which is significantly higher than the $1.4 \%$ found in the study by Kane et $a l$ and the $4.3 \%$ found in the Melles $e t$ al study. Interestingly, the MAE found for the Hill 2.0 in the 'Out of Bounds' group was lower than 'In bounds' group indicating that the boundary model may be imprecise in identifying cases where the calculation is likely to be inaccurate.

The Barrett Universal 2 has been shown to be the most accurate formula in previous IOL power studies; ${ }^{24}$ however, it has never been assessed against the Kane, the Hill 2.0 and only once against the Olsen PO formula. ${ }^{2}$ In our study, the Barrett Universal 2 had a lower MAE than the SRK/T and Hoffer $Q$ which is similar to the findings in other studies. ${ }^{2317}$

The Holladay 2 formula has been updated with a new $\mathrm{AL}$ adjustment (replacing the Wang and Koch adjustment used previously). In this study, it had the fifth lowest MAE and failed to show a statistically significant improvement in accuracy compared with the Holladay 1, SRK/T or the Haigis.

This study follows the recommended method for IOL power studies suggested by Hoffer $e t a l^{18}$ except we used the MAE of formulas to rank their accuracy as suggested by Kane $e t a l^{\beta}$ and Wang et al. ${ }^{14}$ The potential for bias from variation in operative style or refractive technique has been reduced as all patients included were operated on by a single surgeon, using a single IOL model and with relative consistency of staff performing the biometry and postoperative refraction. The high rate of follow-up (only $4.3 \%$ of patients without a postoperative refraction compared with $18.3 \%$ in Melles et al) is another strength of the study.

A potential limitation of the study is the three different IOLMaster models used, although this is unlikely to have affected the outcome as measurements taken between the IOLMaster 500 and 700 have been shown to have excellent agreement. ${ }^{19}$ The IOLmaster models differ in their technique for measurement of anterior chamber depth, Model 500 measures with a slit beam and Model 700 uses swept source optical coherence tomography. Akman identified the Model 700 measured the ACD slightly shorter than the $500 .^{19}$
Another limitation is that not all cases had measurements of lens thickness and CCT. The Olsen and Kane formulas use both LT and CCT as variables and the Holladay 2 and Barrett use LT and WTW as a variable. When these variables were included, all four formulas performed more accurately than when they were not available.

Cases underwent surgery over an 11-year period and biometry performed using three different IOLMaster models (3, 500 and 700). This may have had small impact on our results since the more modern formulas, in general, are more accurate when LT and CCT are available, parameters not measured on the older model 3. This may have had the effect of underestimating the prediction accuracy advantage of the newer formulas.

The numbers in this study were inadequate to perform a meaningful, adequately powered subgroup analysis comparing formula accuracy for short, medium or long axial length eyes. Short eyes remain the group with the highest absolute error and the Kane formula demonstrated no benefit. Similar to the finding of Melles, ${ }^{2}$ the Hoffer $Q$ formula demonstrated no advantage over other earlier generation formulas for short eyes.

With respect to the Haigis formula, we found no additional benefit with optimisation of all three constants over a0 constant optimisation, a finding reported elsewhere. ${ }^{2}$

These results were obtained using lens constants optimised by the surgeon within their own group of patients. For an individual surgeon wanting to achieve similar results, it is important they optimise their own lens constants.

Contributors BC and JK planned the study, analysed the data, drafted, read and approved the manuscript. BC collected the data and submitted the study. BC and JK supervised the study and are its guarantors.

Funding The authors have not declared a specific grant for this research from any funding agency in the public, commercial or not-for-profit sectors.

Competing interests $\mathrm{JK}$ is the owner of the JXK formula.

Patient consent for publication Not required.

Ethics approval Approval was obtained from the Royal Victorian Eye and Ear Hospital Human Research and Ethics Committee.

Provenance and peer review Not commissioned; externally peer reviewed.

Open access This is an open access article distributed in accordance with the Creative Commons Attribution Non Commercial (CC BY-NC 4.0) license, which permits others to distribute, remix, adapt, build upon this work non-commercially, and license their derivative works on different terms, provided the original work is properly cited, appropriate credit is given, any changes made indicated, and the use is non-commercial. See: http://creativecommons.org/licenses/by-nc/4.0/.

\section{REFERENCES}

1. Aristodemou P, Knox Cartwright NE, Sparrow JM, et al. Intraocular lens formula constant optimization and partial coherence interferometry biometry: refractive outcomes in 8108 eyes after cataract surgery. J Cataract Refract Surg 2011;37:50-62.

2. Melles RB, Holladay JT, Chang WJ. Accuracy of intraocular lens calculation formulas. Ophthalmology 2018;125:169-78.

3. Kane JX, Van Heerden A, Atik A, et al. Intraocular lens power formula accuracy: comparison of 7 formulas. J Cataract Refract Surg 2016;42:1490-500.

4. Kane JX, Van Heerden A, Atik A, et al. Accuracy of 3 new methods for intraocular lens power selection. J Cataract Refract Surg 2017;43:333-9. 
5. Haigis W, Lege B, Miller N, et al. Comparison of immersion ultrasound biometry and partial coherence interferometry for intraocular lens calculation according to Haigis. Graefes Arch Clin Exp Ophthalmol 2000;238:765-73.

6. Hoffer KJ. The Hoffer $Q$ formula: a comparison of theoretic and regression formulas. J Cataract Refract Surg 1993;19:700-12.

7. Zuberbuhler B, Morrell AJ. Errata in printed Hoffer Q formula. J Cataract Refract Surg 2007;33.

8. Holladay JT, Prager TC, Chandler TY, et al. A three-part system for refining intraocular lens power calculations. J Cataract Refract Surg 1988;14:17-24.

9. Retzlaff JA, Sanders DR, Kraff MC. Development of the SRK/T intraocular lens implant power calculation formula. J Cataract Refract Surg 1990;16:333-40.

10. Olsen T. PhacoOptics. Available: https://www.phacooptics.net/ [Accessed 22 May 2018].

11. Holladay JT. Holladay IOL consultant software \& surgical outcomes assessment. Bellaire, TX: Holladay Consulting, 2015.
12. Barrett GD. Barrett universal II formula Singapore: Asia-Pacific association of cataract and refractive surgeons. Available: https:// www.apacrs.org/barrett_universal2/ [Accessed 22 May 2017].

13. Hill W. Hill-RBF method. Available: https://rbfcalculator.com/ [Accessed 22 May 2018].

14. Wang L, Koch DD, Hill W, et al. Pursuing perfection in intraocular lens calculations: III. Criteria for analyzing outcomes. J Cataract Refract Surg 2017;43:999-1002.

15. Aristodemou P, Knox Cartwright NE, Sparrow JM, et al. Statistical analysis for studies of intraocular lens formula accuracy. Am J Ophthalmol 2015;160:1085-6.

16. Aristodemou P, Sparrow JM, Kaye S. Evaluating refractive outcomes after cataract surgery. Ophthalmology 2019;126:13-18.

17. Cooke DL, Cooke TL. Comparison of 9 intraocular lens power calculation formulas. J Cataract Refract Surg 2016;42:1157-64.

18. Hoffer KJ, Aramberri J, Haigis W, et al. Protocols for studies of intraocular lens formula accuracy. Am J Ophthalmol 2015;160:403-5.

19. Akman A, Asena L, Güngör SG. Evaluation and comparison of the new swept source OCT-based IOLMaster 700 with the IOLMaster 500. Br J Ophthalmol 2016;100:1201-5. 